Canadian Oncology

Nursing Journal

Revue canadienne

de soins infirmiers

en oncologie

Volume 31, Issue 3 • Summer 2021

elSSN: 2368-8076 


\section{Liens entre les besoins non satisfaits, la qualité de vie et les caractéristiques des survivantes de cancers gynécologiques en Indonésie}

par Yati Afiyanti, Besral Besral, Haryani Haryani, Ariesta Milanti, Lina Anisa Nasution, Kemala Rita Wahidi, Dewi Gayatri

\section{RÉSUMÉ}

Les survivantes de cancers gynécologiques ont des besoins complexes très souvent négligés. La présente étude indonésienne vise à établir les liens entre les besoins non satisfaits, la qualité de vie et certaines caractéristiques particulières de ces survivantes. Au total, 298 participantes ont rempli le questionnaire sur les besoins non satisfaits des survivants au cancer (Cancer Survivor Unmet Needs ou CaSUN), le questionnaire sur la qualité de vie EORTC QLQ-C30 ainsi que les questionnaires démographiques et cliniques. L'étude, réalisée suivant un devis corrélationnel transversal, a établi un lien entre les besoins non satisfaits et la dégradation de la qualité de vie perçue. Plusieurs facteurs sont associés à l'augmentation des besoins insatisfaits : patients jeunes, revenu moindre, niveau d'éducation moyen, diagnostic récent, stade avancé de la maladie, et polythérapie $(p<.05)$. Chez les survivantes indonésiennes d'un cancer gynécologique, le soutien financier (70,5\%) constitue le besoin le plus souvent insatisfait. À la fin des traitements primaires, elles ont besoin de soins globaux et continus pour gérer les problèmes, nouveaux comme anciens, causés par le cancer et le traitement.

\section{AUTEURS}

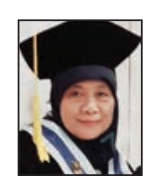

Yati Afiyanti, Faculté de sciences infirmières, Université d'Indonésie (Indonésie), yatiafiyanti@yahoo.com

Besral Besral, Faculté de santé publique, Université d'Indonésie (Indonésie)

Haryani Haryani, Faculté de médecine, de santé publique et de sciences infirmières, École de soins infirmiers, Université Gadjah Mada (Indonésie)

Ariesta Milanti, École de soins infirmiers Nethersole, Université chinoise de Hong Kong (Hong Kong)

Lina Anisa Nasution, Faculté de sciences infirmières, Université d'Indonésie (Indonésie)

Kemala Rita Wahidi, Centre national de cancérologie Dharmais (Indonésie)

Dewi Gayatri, Faculté de sciences infirmières, Université d'Indonésie (Indonésie)

Remerciements : La présente étude a été subventionnée par la Direction de l'enseignement supérieur du ministère de l'Éducation nationale de la République d'Indonésie (No 285/UN2.R3.1/ HKP.05.00/2018).

Conflit d'intérêts : Les auteurs n'ont pas de conflits d'intérêts à déclarer concernant la présente étude.
Mots-clés : cancer, femmes, survivance, qualité de vie, Indonésie

\section{INTRODUCTION}

Les cancers gynécologiques s'attaquent au système reproducteur féminin, c'est-à-dire à l'utérus, au col, aux ovaires, à l'endomètre, aux trompes de Fallope et au vagin (National Cancer Institute, 2019). De tous les cancers féminins, le cancer du col occupe le quatrième rang en matière de fréquence. Selon les estimations, il y a eu, en 2018, 570000 cas et 311000 décès dans le monde (Bray et al., 2018). La majorité des cas sont recensés en Afrique subsaharienne et en Asie du SudEst. C'est en Asie occidentale, notamment en Arabie saoudite et en Irak, que l'incidence du cancer du col est la plus faible, tandis que certains pays d'Europe de l'Est et d'Asie centrale constatent une tendance à la hausse (Bray et al., 2018).

En Indonésie, le cancer du col est le deuxième en importance chez les femmes après le cancer du sein (ministère de la Santé de la République d'Indonésie, 2019b). On estime son incidence à 23,4 femmes sur 100 000, et le taux de mortalité à 13,9 sur 100000 (ministère de la Santé de la République d'Indonésie, 2019b). Toujours en Indonésie, on estime aussi que les autres types de tumeurs gynécologiques, comme le cancer de l'utérus ou des ovaires, touchent respectivement 8 et 6 femmes par 100000 (ministère de la Santé de la République d'Indonésie, 2015).

Les preuves sont là : améliorer le dépistage et le traitement du cancer a contribué à la hausse du nombre de survivants, y compris pour les cancers gynécologiques (Schlumbrecht, Sun, Huang, Milbourne et Bodurka, 2018). Les survivantes de ces cancers forment un groupe à part, car le traitement cause des effets secondaires et des problèmes particuliers pour la qualité de vie, comme la dysfonction sexuelle et les fractures par insuffisance osseuse de la ceinture pelvienne (Schmeler et al., 2010; Shih et al., 2013). Pourtant, ces besoins complexes sont trop souvent négligés (Rosenberg et Partridge, 2017). Pour améliorer l'état de santé des survivantes à court et à long terme, il faut comprendre les défis individuels et les besoins non satisfaits; c'est la clé pour adapter au mieux les interventions (Rosenberg et Partridge, 2017).

Les soins aux survivants sont devenus la norme dans de nombreux établissements disposant de ressources suffisantes pour offrir ces services (Fitch, 2014; Nekhlyudov, Ganz, Arora et Rowland, 2017; Rosenberg et Partridge, 2017; Salani, Khanna, Frimer, Bristow et Chen, 2017). Une étude menée dans dix pays de l'Asie du Pacifique (Chine, Japon, RAS de Hong Kong, Corée du Sud, Myanmar, Thaïlande, Inde, Singapour, Philippines et Australie) a mis en lumière l'écart 
dans les services aux survivants entre les pays à revenu élevé et ceux à revenu relativement faible et intermédiaire (Molassiotis et al., 2017). L'Indonésie travaille actuellement à mettre en place un programme de soins pour les survivantes de cancers gynécologiques. La présente étude s'est penchée sur les besoins insatisfaits, la qualité de vie (QV) perçue et les liens entre ces variables afin d'orienter les politiques et pratiques encadrant les soins prodigués à ce groupe particulier.

\section{MÉTHODOLOGIE}

Une étude transversale de patientes atteintes d'un cancer gynécologique a été menée dans trois grands hôpitaux de recours situés dans les provinces indonésiennes de Jakarta, Yogyakarta et de Java-Est. Le recrutement des participantes a duré 3 mois.

Léchantillon de commodité se composait de patientes adultes (plus de 18 ans) atteintes d'un cancer gynécologique et ayant terminé leur traitement primaire depuis au moins un mois. Étaient exclues les patientes ayant subi une récidive ou souffrant d'un trouble cognitif les empêchant de participer à l'étude. Dans les établissements choisis pour l'étude, les infirmières travaillant dans les unités de soins ambulatoires ont participé au recrutement des participantes ainsi qu'à la collecte de données à l'aide de questionnaires sur papier. Au total, 310 patientes ont été approchées et 298 ont accepté de participer (taux de réponse $=96,13 \%$ ).

Les participantes ont rempli un questionnaire sociodémographique, version indonésienne du questionnaire CaSUN (Cancer Survivors' Unmet Needs) (Hodgkinson et al., 2007a), et le questionnaire EORTC QLQ-C30 (Aaronson et al., 1993; Perwitasari et al., 2011). Le questionnaire sociodémographique, conçu par les auteurs, visait à recueillir des données sur l'âge, l'état civil, le niveau de scolarité, le statut d'emploi et le revenu familial mensuel des participantes. Des données cliniques ont aussi été prélevées dans leur dossier médical : temps écoulé depuis le diagnostic, stade de la maladie, présence de métastases et type de traitement primaire reçu.

Le questionnaire CaSUN, qui comprend 35 items, divise les besoins des survivants en quatre catégories : 1) besoins existentiels, 2) soins globaux, 3) information, 4) qualité de vie, et 5) relations (Hodgkinson et al., 2007a). Dans le questionnaire CaSUN, les items reçoivent une note différente selon que le besoin est satisfait ou non; par la suite, on calcule le total des besoins, ainsi que l'intensité. Autrement dit, les répondants précisent si l'item constitue un besoin pour eux ou s'il ne s'applique pas à leur situation, et si ce besoin est satisfait ou non. L'intensité du besoin non satisfait peut être faible (1 point), modérée ( 2 points) ou forte ( 3 points). Le score total s'obtient en additionnant les points pour l'ensemble des 35 items (Hodgkinson et al., 2007a). Dans de nombreux pays, cet outil s'est révélé fiable pour évaluer les besoins non satisfaits des survivants à différents cancers (Bender et al., 2012; Molassiotis et al., 2017; Smith et al., 2013). La version indonésienne montre également que la validité et la fiabilité sont bonnes (alpha de Cronbach $=0,75-0,95)$.

Le questionnaire EORTC QLQ-C30 mesure différents aspects de la qualité de vie $(\mathrm{QV})$ perçue par les patients cancéreux : le rôle, les dimensions physique, cognitive, émotionnelle et sociale, de même que les répercussions financières du cancer (Aaronson et al., 1993). Le questionnaire EORTC QLQ-C30 utilise quant à lui cinq échelles fonctionnelles, trois échelles de symptômes, une échelle mesurant l'état de santé et la QV globales, ainsi que six items indépendants. Toutes les échelles et tous les items reçoivent un score de 0 à 100. Un score élevé sur une échelle dénote un haut niveau de fonctionnement; par exemple, si le score d'une échelle fonctionnelle est élevé, le niveau de fonctionnement est très bon (Aaronson et al., 1993). Le questionnaire EORTC QLQC30 est largement reconnu comme un outil valide et fiable pour mesurer la qualité de vie des patients atteints de cancer, même en Indonésie (Perwitasari et al., 2011).

Une fois les questionnaires remis, on a écarté ceux qui étaient incomplets. Seuls les questionnaires entièrement remplis ont été conservés pour l'analyse des données. Les caractéristiques démographiques et cliniques, ainsi que les besoins non satisfaits (CASUN), et la qualité de vie (EORTC QLQ-C30) des participantes ont été analysés à l'aide de statistiques descriptives (moyenne, médiane, écart type, pourcentage, minimum et maximum). Le score total des besoins insatisfaits correspondait à la somme des points (de 1 à 3) pour les 35 items du questionnaire CASUN. Au score total de QV (EORTC QLQ-C30), qui correspond à la moyenne des items (score brut), on a appliqué une transformation linéaire pour obtenir une note sur une échelle de 0 à 100 et ensuite procéder à des analyses bivariées et multivariées afin d'établir le lien entre le score total des besoins non satisfaits et de la QV pour une valeur $\mathrm{p}$ de .05. Les analyses statistiques ont été réalisées à l'aide de la version 22 du logiciel SPSS (SPSS Inc., Chicago, Illinois, États-Unis).

Létude a été approuvée par le comité d'éthique de la Faculté de médecine de l'Université Gadjah Mada et par la Faculté de sciences infirmières de l'Université d'Indonésie (numéro de référence : 28/UN2.F12.D/HKP.02.04/2018). Toutes les participantes éventuelles ont reçu l'information de base sur l'étude et celles qui ont accepté d'y participer devaient, avant d'être admises, fournir un consentement éclairé.

\section{RÉSULTATS}

En tout, 298 participantes sur 310 ont rempli les questionnaires en entier. La moyenne d'âge était de 50,3 ans (ÉT =9,6). La plupart des participantes étaient mariées (88,3\%) et avaient fréquenté l'école primaire et secondaire. Près de la moitié $(48,7 \%)$ étaient femmes au foyer et avaient une situation économique précaire, avec un revenu familial inférieur à 71 \$US par mois. Plus du tiers des participantes (37,6 \%) souffraient d'un cancer gynécologique de stade III et la moitié avaient reçu de la chimiothérapie (voir tableau 1).

\section{Besoins non satisfaits}

Chez les survivantes de cancers gynécologiques, le besoin insatisfait le plus fréquemment rapporté était le soutien financier (70,5 \%). À l'opposé, le besoin le moins fréquent était l'aide pour s'adapter aux changements corporels - seulement 29,9\% des survivantes ont mentionné que ce besoin n'était 
pas satisfait, et $43 \%$ ne le ressentaient pas du tout. Plus de la moitié des participantes ont dit se sentir en confiance dans les communications avec de l'équipe de soins (à l'écoute des difficultés); pour elles, ce besoin était comblé. En ce qui concerne les scores totaux, les trois catégories de besoins non satisfaits les plus importantes étaient les soins globaux $(96,6 \%)$, les besoins existentiels $(79 \%)$ et l'information (62\%) (voir le tableau 2).

Selon les analyses, les patientes plus jeunes, les femmes à faible revenu et celles ayant un meilleur niveau de scolarité affichaient davantage de besoins non satisfaits (scores totaux élevés). En ce qui concerne le profil clinique, les patientes ayant reçu une polythérapie et dont le diagnostic était plus récent et le cancer plus avancé rapportaient aussi plus de besoins insatisfaits (scores totaux plus élevés) que celles qui n'avaient reçu qu'une seule forme de traitement primaire, dont le diagnostic était moins récent ou le cancer moins avancé (voir le tableau 3).

\section{Qualité de vie}

Le score total de qualité de vie $(\mathrm{QV})$ est corrélé négativement avec le score total des besoins insatisfaits $(p=.000)$ (voir le tableau 4). Par contraste, les six variables liées aux symptômes (c.-à-d. fatigue, nausées et vomissements, douleur, perte d'appétit, constipation et difficultés financières) sont corrélées positivement avec les besoins insatisfaits $(p<.05)$, ce qui signifie que les survivantes de cancers gynécologiques qui ont une moins bonne qualité de vie perçue et qui éprouvent des symptômes plus intenses ont davantage de besoins non satisfaits. On a également constaté un lien significatif entre les besoins non satisfaits et dix des quinze domaines relatifs à la qualité de vie $(\mathrm{QV})$. De plus, quatre variables (santé générale, fonctionnement physique, fonctionnement émotionnel et fonctionnement cognitif) étaient négativement corrélées avec les besoins insatisfaits. Les participantes ayant un meilleur état de santé général, fonctionnement physique, fonctionnement émotionnel et fonctionnement cognitif rapportaient moins de besoins non satisfaits. Les trois symptômes les plus fréquents étaient l'insomnie $(43,2 \%)$, les difficultés financières $(38,8 \%)$ et la douleur $(36,8 \%)$.

\section{DISCUSSION}

Notre principal constat : plus il y a de besoins non satisfaits, plus la QV perçue est faible. Ces observations concordent avec celles d'études antérieures réalisées auprès de patientes indonésiennes atteintes d'un cancer gynécologique en traitement actif (Putri, Afiyanti, Ungsianik et Milanti, 2017) et de survivantes australiennes de cancers gynécologiques en post-traitement (Hodgkinson et al., 2007b; Urbaniec, Collins, Denson et Whitford, 2011). D'autres études portant sur la survivance à différents types de cancers (Molassiotis et al., 2017; So et al., 2013) et au cancer du sein (So et al., 2014) ont également produit des résultats similaires.

Nos résultats montrent que les besoins non satisfaits ont des répercussions négatives sur la qualité de vie. Cette situation serait attribuable au fait qu'en Indonésie, les survivants au cancer ne reçoivent qu'un minimum de soins. Ils se rendent
Tableau 1. Caractéristiques des participantes $(n=298)$

\begin{tabular}{|c|c|}
\hline Caractéristiques & Moyenne (ÉT) \\
\hline Âge (ans) & $\begin{array}{c}\text { 50,3 (ÉT 9,6; écart } \\
\text { 20-71) }\end{array}$ \\
\hline État civil & $f(\%)$ \\
\hline Mariée & $263(88,3)$ \\
\hline Célibataire & $12(4,0)$ \\
\hline Veuve & $23(7,7)$ \\
\hline \multicolumn{2}{|l|}{ Niveau de scolarité } \\
\hline Non scolarisée & $20(6,7)$ \\
\hline École primaire & $106(35,6)$ \\
\hline École secondaire de premier cycle & $54(18,1)$ \\
\hline École secondaire de deuxième cycle & $90(30,2)$ \\
\hline Collège/université & $28(9,4)$ \\
\hline \multicolumn{2}{|l|}{ Revenu familial mensuel (approx. en \$US) } \\
\hline$<71$ & $143(48,0)$ \\
\hline $71-141$ & $75(25,2)$ \\
\hline $141-212$ & $28(9,4)$ \\
\hline $212-282$ & $23(7,7)$ \\
\hline $282-353$ & $8(2,7)$ \\
\hline$>353$ & $21(7,0)$ \\
\hline \multicolumn{2}{|l|}{ Emploi } \\
\hline Femme au foyer & $145(48,7)$ \\
\hline Fonctionnaire & $11(3,7)$ \\
\hline Employée privée & $27(9,1)$ \\
\hline Travailleuse autonome & $59(19,8)$ \\
\hline Ouvrière d'usine & $44(14,8)$ \\
\hline À la retraite & $12(4,0)$ \\
\hline \multicolumn{2}{|l|}{ Stade du cancer } \\
\hline 1 & $54(18,1)$ \\
\hline II & $86(28,9)$ \\
\hline III & $112(37,6)$ \\
\hline IV & $13(4,4)$ \\
\hline Inconnu & $33(11,1)$ \\
\hline \multicolumn{2}{|l|}{ Temps écoulé depuis le diagnostic } \\
\hline$<1$ an & $110(36,9)$ \\
\hline$\geq 1$ an, $<2$ ans & $124(41,6)$ \\
\hline$\geq 2$ ans, $<4$ ans & $35(11,7)$ \\
\hline$\geq 4$ ans & $29(9,8)$ \\
\hline \multicolumn{2}{|l|}{ Cancer métastatique } \\
\hline Oui & $57(19,2)$ \\
\hline Non & $241(80,9)$ \\
\hline \multicolumn{2}{|l|}{ Traitement primaire } \\
\hline Chimiothérapie & $149(50)$ \\
\hline Chimiothérapie et radiothérapie & $49(16,4)$ \\
\hline Radiothérapie & $38(12,8)$ \\
\hline Chirurgie & $26(8,7)$ \\
\hline Autre & $36(12,1)$ \\
\hline
\end{tabular}




\begin{tabular}{|c|c|c|c|}
\hline Besoins des survivantes - items individuels & Pas un besoin & Besoin satisfait & Besoin non satisfait \\
\hline \multicolumn{4}{|l|}{ Besoins existentiels ( 14 items) } \\
\hline 10 Réduire le stress & 29,9 & 29,9 & 40,3 \\
\hline 19 Apaiser mes inquiétudes concernant la récidive & 16,4 & 27,5 & 56,0 \\
\hline 20 Trouver du soutien émotionnel & 8,1 & 56,0 & 35,9 \\
\hline 23 Rebâtir ma relation avec mon partenaire & 36,9 & 22,5 & 40,6 \\
\hline 24 Discuter avec d'autres patients atteints de cancer & 45,3 & 17,8 & 36,9 \\
\hline 25 Expliquer le cancer aux autres & 12,8 & 46,3 & 40,9 \\
\hline 26 M'adapter aux changements corporels & 43,0 & 27,2 & 29,9 \\
\hline 29 Tourner la page sur le cancer & 7,0 & 49,0 & 44,0 \\
\hline 30 Renforcer mes croyances & 10,1 & 53,0 & 36,9 \\
\hline 31 Reconnaître les répercussions de la maladie & 39,9 & 23,2 & 36,9 \\
\hline 32 Savoir à quoi m'attendre comme survivante & 16,4 & 44,0 & 39,6 \\
\hline 33 Prendre des décisions concernant l'avenir & 12,8 & 51,7 & 35,6 \\
\hline 34 Renforcer ma foi & 6,7 & 54,0 & 39,3 \\
\hline 35 Donner une valeur à ma vie & 6,7 & 49,3 & 44,0 \\
\hline \multicolumn{4}{|l|}{ Soins globaux } \\
\hline 4 Obtenir les meilleurs soins médicaux & 1,7 & 57,0 & 41,3 \\
\hline 5 Avoir accès à des soins de santé locaux & 11,7 & 35,6 & 52,7 \\
\hline 6 Collaborer avec l'équipe de soins & 4,7 & 59,7 & 35,6 \\
\hline 7 Savoir que l'équipe de soins travaille de concert & 2,7 & 62,8 & 34,6 \\
\hline 8 Me faire entendre et me faire prendre au sérieux quand je signale une difficulté & 2,3 & 55,0 & 42,6 \\
\hline 18 Stationnement accessible à l'hôpital & 31,9 & 31,5 & 36,6 \\
\hline \multicolumn{4}{|l|}{ Information (3 items) } \\
\hline 1 Information à jour & 2,7 & 45,3 & 52,0 \\
\hline 2 Information pour les membres de la famille & 4,0 & 54,4 & 41,6 \\
\hline 3 Information facile à comprendre & 1,3 & 54,7 & 44,0 \\
\hline \multicolumn{4}{|l|}{ Qualité de vie } \\
\hline 11 Contrôle des effets secondaires & 13,4 & 38,3 & 48,3 \\
\hline 12 Changements dans la qualité de vie & 18,1 & 41,3 & 40,6 \\
\hline \multicolumn{4}{|l|}{ Relations (3 items) } \\
\hline 21 Soutien au partenaire/ à la famille & 19,5 & 45,3 & 35,2 \\
\hline 22 Gestion des répercussions du cancer sur ma relation de couple & 36,9 & 22,5 & 40,6 \\
\hline 27 Problèmes sexuels & 52,0 & 10,4 & 37,6 \\
\hline \multicolumn{4}{|l|}{ Autre } \\
\hline 9 Information sur les traitements complémentaires & 54,0 & 10,7 & 35,2 \\
\hline 15 Soutien financier et prestations gouvernementales & 13,1 & 16,4 & 70,5 \\
\hline 17 Aide pour l'accès aux services juridiques & 21,8 & 12,4 & 65,8 \\
\hline 14 Aide au maintien de l'emploi & 33,6 & 30,2 & 36,2 \\
\hline
\end{tabular}




\begin{tabular}{|c|c|c|c|c|}
\hline \multicolumn{5}{|c|}{$\begin{array}{l}\text { Tableau 3. Liens entre les caractéristiques des participantes et les } \\
\text { besoins insatisfaits }\end{array}$} \\
\hline \multirow[t]{2}{*}{ Variable } & \multicolumn{2}{|c|}{ Modèle complet } & \multicolumn{2}{|c|}{ Modèle final } \\
\hline & B & Sig. & B & Sig. \\
\hline Constante & 22,3 & 0,000 & 23,3 & 0,000 \\
\hline Âge (an) & $-0,2$ & 0,010 & $-0,2$ & 0,006 \\
\hline Revenu (rupiah) & $-1,4$ & 0,003 & $-1,5$ & 0,002 \\
\hline $\begin{array}{l}\text { Temps écoulé depuis } \\
\text { le diagnostic (an) }\end{array}$ & $-1,6$ & 0,007 & $-1,6$ & 0,005 \\
\hline \multicolumn{5}{|l|}{$\begin{array}{l}\text { Éducation École } \\
\text { primaire (réf.) }\end{array}$} \\
\hline $\begin{array}{l}\text { École secondaire } \\
\text { de premier cycle }\end{array}$ & 3,1 & 0,065 & 3,0 & 0,066 \\
\hline $\begin{array}{l}\text { École secondaire } \\
\text { de deuxième } \\
\text { cycle }\end{array}$ & 2,5 & 0,123 & 2,4 & 0,127 \\
\hline $\begin{array}{l}\text { Collège/ } \\
\text { université }\end{array}$ & 4,6 & 0,068 & 4,1 & 0,091 \\
\hline \multicolumn{5}{|l|}{$\begin{array}{l}\text { Stade du cancer } \\
\text { I (réf.) }\end{array}$} \\
\hline ॥ & 3,2 & 0,069 & 3,1 & 0,079 \\
\hline III & 7,2 & 0,000 & 7,0 & 0,000 \\
\hline IV & 3,2 & 0,127 & 2,8 & 0,158 \\
\hline \multicolumn{5}{|l|}{$\begin{array}{l}\text { Traitement primaire } \\
\text { Radiothérapie (réf.) }\end{array}$} \\
\hline $\begin{array}{l}\text { Chimio- } \\
\text { radiothérapie }\end{array}$ & 2,9 & 0,040 & 3,1 & 0,028 \\
\hline Chirurgie & $-2,5$ & 0,112 & $-2,4$ & 0,121 \\
\hline Métastases & $-1,3$ & 0,421 & & NS \\
\hline État civil & $-0,4$ & 0,836 & & NS \\
\hline \multicolumn{5}{|l|}{$\begin{array}{l}\text { Profession } \\
\text { Fonctionnaire (réf.) }\end{array}$} \\
\hline $\begin{array}{l}\text { Employée d'une } \\
\text { entreprise privée }\end{array}$ & 1,1 & 0,532 & & NS \\
\hline Femme au foyer & 0,9 & 0,551 & & NS \\
\hline
\end{tabular}

Tableau 4. Liens entre la qualité de vie et les besoins insatisfaits $(n=298)$

\begin{tabular}{|c|c|c|}
\hline Variable & Analyses & \\
\hline & $\begin{array}{l}\text { Moyenne } \\
\text { (ÉT) }\end{array}$ & $\begin{array}{c}\text { valeur } p \\
\text { (coefficient de } \\
\text { corrélation) }\end{array}$ \\
\hline Qualité de vie (score total) & $76,4(16,5)$ & $0,000 *(-0,253)$ \\
\hline État de santé général & $67,0(20,1)$ & $0,000 *(-0,345)$ \\
\hline \multicolumn{3}{|l|}{ Aspect fonctionnel } \\
\hline Fonctionnement physique & $75,9(22,9)$ & $0,015^{*}(-0,142)$ \\
\hline Rôle & $73,8(29,8)$ & $0,071(-0,105)$ \\
\hline Fonctionnement émotionnel & $80,5(20,9)$ & $0,000^{*}(-0,241)$ \\
\hline Fonctionnement cognitif & $84,5(20,3)$ & $0,000 *(-0,273)$ \\
\hline Fonctionnement social & $81,5(23,3)$ & $0,124(-0,090)$ \\
\hline \multicolumn{3}{|l|}{ Symptôme } \\
\hline Fatigue & $35,7(26,2)$ & $0,008^{*}(0,155)$ \\
\hline Nausées/vomissement & $16,4(25,6)$ & $0,006^{*}(0,160)$ \\
\hline Douleur & $32,6(30,5)$ & $0,001^{*}(0,191)$ \\
\hline Dyspnée & $8,1(19,5)$ & $0,167(0,081)$ \\
\hline Insomnie & $30,2(34,2)$ & $0,507(0,039)$ \\
\hline Perte d'appétit & $29,6(34,5)$ & $0,000 *(0,212)$ \\
\hline Constipation & $17,4(29,2)$ & $0,029^{*}(0,127)$ \\
\hline Diarrhée & $8,9(21,3)$ & $0,219(0,072)$ \\
\hline Difficultés financières & $39,0(36,4)$ & $0,001^{*}(0,197)$ \\
\hline
\end{tabular}

habituellement à des rendez-vous de suivi en consultation externe où l'on vérifie leur état de santé et l'absence de récidive de la maladie. Toutefois, la continuité des soins infirmiers est très mauvaise.

En revanche, dans la présente étude, la plupart des survivantes n'ont rapporté aucune insatisfaction quant aux soins psychosociaux ou à l'information, contrairement aux autres études menées en Occident et à Hong Kong (Armes et al., 2009; Knobf et al., 2012; So et al., 2013). Peut-être ne savaientelles pas qu'il existe des soins pour les survivants, alors elles n'en ont pas demandé. Elles étaient davantage préoccupées par les difficultés financières.

Dans la présente étude, le plus gros problème rencontré par les survivantes indonésiennes de cancers gynécologiques était de nature financière. Non seulement il s'agissait du plus important besoin non satisfait, mais cétait aussi le second point en importance (après l'insomnie) mentionné par les survivantes dans leur expérience des symptômes. Plus de $70 \%$ des participantes vivaient avec un revenu mensuel familial moyen inférieur à 141 \$US. Ce montant est même 
inférieur au coût de la vie pour une personne selon les estimations du salaire minimum régional (qui varie selon les différents milieux où l'étude a été réalisée; Jakarta $=\sim 281$ \$US, Surabaya $=\sim 276$ \$US, Yogyakarta $=\sim 112 \$$ US). Toutefois, nous ignorons si le revenu familial a changé entre le moment du diagnostic et du traitement, la productivité des patientes pouvant avoir été limitée par la maladie.

Ces résultats sont le signe d'une toxicité financière, terme qui désigne la précarité et la détresse financière subjective due au cancer et au coût des traitements (Ratain, 2009; Yabroff, Lawrence, Clauser, Davis et Brown, 2004). De plus en plus, on considère la toxicité financière comme un lourd et inquiétant fardeau pour les survivants du cancer, particulièrement aux États-Unis, où les coûts des traitements, y compris les dépenses personnelles associées, continuent d'augmenter (Altice, Banegas, Tucker-Seeley et Yabroff, 2017; Hastert et al., 2018; Yabroff et al., 2004). En Indonésie, il y a très peu d'études sur la toxicité financière. Selon un sondage à petite échelle tenu à Jakarta, les survivants du cancer, même ceux qui détiennent une assurance maladie, seraient touchés par la toxicité financière (Pangestu et Karnadi, 2018).

Dans une étude indonésienne de 2014 portant sur les besoins insatisfaits de patients cancéreux de stade avancé, on soutient qu'il pas surprenant que de nombreux patients rapportent des besoins financiers étant donné la grande proportion (3/4) de la population ne disposant pas d'une assurance adéquate (Effendy et al., 2014). Toutefois, depuis 2014, le gouvernement indonésien a créé une assurance maladie nationale qui inclut les traitements du cancer (Agustina et al., 2019). Ce régime offre une couverture complète des traitements du cancer, bien que certains médicaments soient exclus (ex. bevacizumab et cétuximab) (ministère de la Santé de la République d'Indonésie, 2019a). Mais même dans les pays d'Asie du SudEst disposant d'un régime universel de soins de santé comme la Malaisie, les dépenses personnelles associées au traitement représentent tout de même une charge très lourde pour les patients (Tangcharoensathien et al., 2011).

Pour les survivants du cancer, l'ampleur des difficultés financières englobe les dépenses personnelles de nature médicale ou non médicale (ex. transport), les coûts indirects dus à la perte de revenus, ainsi que les dettes et les faillites causées par les traitements (Altice et al., 2017). La plupart des participantes à l'étude étaient avaient été dirigées vers l'établissement de santé par des médecins de différentes régions de l'Indonésie; par conséquent, le transport était dispendieux. Selon la coutume indonésienne, les patients sont toujours accompagnés par des proches, souvent le mari dans le cas des patientes souffrant d'un cancer gynécologique. Par conséquent, ce proche aidant doit parfois quitter son emploi malgré les dépenses supplémentaires qu'entraînent le traitement du cancer et les rendez-vous de suivi (Anggraeni et Ekowati, 2011; Kristanti, Setiyarini et Effendy, 2017). D’autres études seront nécessaires pour examiner la toxicité financière, sa source et ses répercussions sur les patientes indonésiennes atteintes d'un cancer gynécologique et sur les proches qui s'occupent d'elles.

Pour les familles pauvres, le cancer peut avoir des conséquences encore plus désastreuses (Kimman et al., 2015). La présente étude a aussi établi des liens entre un faible revenu familial, des niveaux élevés de besoins non satisfaits et une piètre qualité de vie, confirmant ainsi les résultats de nombreuses études antérieures réalisées tant dans les pays à revenu élevé que dans les pays à revenu relativement faible et à revenu intermédiaire (Chang et al., 2014; Kimman et al., 2017; Molassiotis et al., 2017; Rahman, Ahsan, Monalisa et Rahman, 2014; Sleight, Lyons, Vigen, Macdonald, et Clark, 2018). Les patients de milieux socioéconomiques défavorisés ont parfois plus de bâtons dans les roues pour accéder aux services de santé, aux traitements du cancer et au suivi (DiMartino, Birken et Mayer, 2017; Niksic et al., 2015). En Indonésie, les coûts médicaux indirects, qui ne sont pas couverts par les assurances, ainsi que les procédures d'aiguillage complexes et chronophages sont reconnus comme les principales contraintes d'accès aux services de santé pour les patients cancéreux (Fles et al., 2017).

En outre, la présente étude montre que certains groupes de survivantes de cancers gynécologiques ont besoin de plus de soutien pour améliorer leur qualité de vie. Nos résultats confirment ceux des études antérieures selon lesquels les survivants dont le traitement vient de se terminer, de même que ceux dont la maladie est plus avancée, qui reçoivent une polythérapie ou qui sont plus jeunes nécessitent une attention particulière (Hodgkinson et al., 2007b; Kimman et al., 2017; Molassiotis et al., 2017; Urbaniec et al., 2011). Par exemple, les femmes plus jeunes s'inquiètent de leur fertilité et de leur fonction reproductive.

\section{LIMITES}

Les conclusions de la présente étude doivent être interprétées avec prudence. Bien qu'il s'agisse de la première étude multicentrique se penchant sur les besoins non satisfaits des survivantes de cancers gynécologiques en Indonésie, l'échantillon de commodité de taille relativement faible nous empêche de généraliser les résultats à grande échelle. Le devis transversal de l'étude et l'inclusion à la fois de survivantes tout juste sorties de traitement et de survivantes ayant terminé leur traitement depuis longtemps entraînent également leur part de réserve, sachant que le temps est un élément déterminant lorsqu'il est question de survie au cancer (Broom, Kenny et Kirby, 2017). Les besoins et la qualité de vie des survivantes peuvent changer tout au long de leur expérience de la maladie (Kimman et al., 2017).

\section{CONCLUSION}

La présente étude, qui porte un regard nouveau sur les survivantes de cancers gynécologiques, s'ajoute à la recherche montrant le lien existant entre un taux élevé de besoins insatisfaits et une mauvaise qualité de vie en matière de santé. Nos conclusions devraient inciter le gouvernement à offrir un filet de sécurité socio-économique aux gens atteints de cancer et à leurs familles. Elles aideront aussi à créer des services pour les survivants, services qui n'existent pas encore en Indonésie. 


\section{RÉFÉRENCES}

Aaronson, N. K., Ahmedzai, S., Bergman, B., Bullinger, M., Cull, A., Duez, N. J., ... Takeda, F. (1993). The European Organisation for Research and Treatment of Cancer QLQ-C30: A quality-oflife instrument for use in international clinical trials in oncology. Journal of the National Cancer Institute, 85, 365-376. https://doi. org/10.1093/jnci/85.5.365

Agustina, R., Dartanto, T., Sitompul, R., Susiloretni, K. A., Suparmi, Achadi, E. L., ... Khusun, H. (2019). Universal health coverage in Indonesia: concept, progress, and challenges. Lancet, 393(10166), 75-102. https://doi.org/10.1016/S0140-6736(18)31647-7

Altice, C. K., Banegas, M. P., Tucker-Seeley, R. D., \& Yabroff, K. R. (2017). Financial hardships experienced by cancer survivors: A systematic review. Journal of the National Cancer Institute, 109(2), 1-17. https://doi.org/10.1093/jnci/djw205

Anggraeni, M., \& Ekowati, W. (2011). Family role in the achievement of post radical mastectomy self integrity patients. International Journal of Public Health Research, 1, 163-168.

Armes, J., Crowe, M., Colbourne, L., Morgan, H., Murrells, T., Oakley, C., ... Richardson, A. (2009). Patients' supportive care needs beyond the end of cancer treatment: A prospective, longitudinal survey. Journal of Clinical Oncology, 27(36), 6172-6179. https://doi. org/10.1200/JCO.2009.22.5151

Bender, J. L., Wiljer, D., To, M. J., Bedard, P. L., Chung, P., Jewett, M. A. S., ... Gospodarowicz, M. (2012). Testicular cancer survivors' supportive care needs and use of online support: A cross-sectional survey. Supportive Care in Cancer, 20(11), 2737-2746. https://doi. org/10.1007/s00520-012-1395-x

Bray, F., Jacques, F., Isabelle, S., Rebecca L., S., Lindsey A., T., Ahmedin, J., \& 1. (2018). Global Cancer Statistics 2018: GLOBOCAN Estimates of Incidence and Mortality Worldwide for 36 Cancers in 185 Countries. CA Cancer J Clin, 0, 1-31. https://doi. org/10.3322/caac.21492

Broom, A., Kenny, K., \& Kirby, E. (2017). On waiting, hauntings and surviving: Chronicling life with cancer through solicited diaries. https://doi.org/10.1177/0038026117719216

Chang, O., Choi, E. K., Kim, I. R., Nam, S. J., Lee, J. E., Lee, S. K., ... Cho, J. (2014). Association between socioeconomic status and altered appearance distress, body image, and quality of life among breast cancer patients. Asian Pacific Journal of Cancer Prevention. https://doi.org/10.7314/APJCP.2014.15.20.8607

DiMartino, L. D., Birken, S. A., \& Mayer, D. K. (2017). The Relationship Between Cancer Survivors' Socioeconomic Status and Reports of Follow-up Care Discussions with Providers. Journal of Cancer Education: The Official Journal of the American Association for Cancer Education, 32(4), 749-755. https://doi.org/10.1007/ s13187-016-1024-3

Effendy, C., Vissers, K., Osse, B. H. P., Tejawinata, S., Vernooij-Dassen, M., \& Engels, Y. (2014). Comparison of problems and unmet needs of patients with advanced cancer in a european country and an asian country. Pain Practice. https://doi.org/10.1111/papr.12196

Fitch, M. I. (2014). Supportive care framework: Theoretical underpinnings. In M. I. Fitch (Ed.), Supportive care framework: A foundation for person-centred care. Ontario: CANO/ACIO.

Fles, R., Bos, A. C. R. K., Supriyati, Rachmawati, D., Waliyanti, E., Tan, I. B., ... Dewi, F. S. T. (2017). The role of Indonesian patients' health behaviors in delaying the diagnosis of nasopharyngeal carcinoma. BMC Public Health. https://doi.org/10.1186/s12889-017-4429-y

Hastert, T. A., Young, G. S., Pennell, M. L., Padamsee, T., Zafar, S. Y., DeGraffinreid, C., ... Paskett, E. D. (2018). Financial burden among older, long-term cancer survivors: Results from the LILAC study. Cancer Medicine, 7(9), 4261-4272. https://doi.org/10.1002/ cam4.1671

Hodgkinson, K, Butow, P., Hunt, G. E., Pendlebury, S., Hobbs, K. M., Lo, S. K., \& Wain, G. (2007a). The development and evaluation of a measure to assess cancer survivors' unmet supportive care needs: The CaSUN (Cancer Survivors' Unmet Needs measure). PsychoOncology, Vol. 16, pp. 796-804. https://doi.org/10.1002/pon.1137

Hodgkinson, Katharine, Butow, P., Fuchs, A., Hunt, G. E., Stenlake, A., Hobbs, K. M., ... Wain, G. (2007b). Long-term survival from gynecologic cancer: Psychosocial outcomes, supportive care needs and positive outcomes. Gynecologic Oncology, 104(2), 381-389. https://doi.org/10.1016/j.ygyno.2006.08.036

Kimman, M., Jan, S., Yip, C., Thabrany, H., Peters, S., Bhoo-Pathy, N., ... Klinwimol, T. (2015). Catastrophic health expenditure and 12-month mortality associated with cancer in Southeast Asia: Results from a longitudinal study in eight countries. BMC Medicine.

Kimman, M. L., Jan, S., Peters, S. A. E., Yip, C. H., Ngelangel, C. A., Bhoo-Pathy, N., \& Woodward, M. (2017). Health-related quality of life and psychological distress among cancer survivors in Southeast Asia: results from a longitudinal study in eight low- and middle-income countries. BMC Medicine, 15(1), 10. https://doi. org/10.1186/s12916-016-0768-2

Knobf, M. T., Ferrucci, L. M., Cartmel, B., Jones, B. A., Stevens, D., Smith, M., ... Mowad, L. (2012). Needs assessment of cancer survivors in Connecticut. Journal of Cancer Survivorship, 6(1), 1-10. https://doi.org/10.1007/s11764-011-0198-2

Kristanti, M. S., Setiyarini, S., \& Effendy, C. (2017). Enhancing the quality of life for palliative care cancer patients in Indonesia through family caregivers: a pilot study of basic skills training. BMC Palliative Care, 16(1), 4. https://doi.org/10.1186/ s12904-016-0178-4

Ministry of Health Republic of Indonesia. (2015). Data and information on cancer situation (Data dan Informasi Kesehatan Situasi Penyakit Kanker). Buletin Kanker, 1(1), 1-5. https://doi. org/10.1007/s13398-014-0173-7.2

Ministry of Health Republic of Indonesia. Ministry of Health Decree number HK.01.07/MENKES/707/2018 concerning National Formularium. , (2019).

Ministry of Health Republic of Indonesia. (2019b). World Cancer Day 2019. Retrieved July 16, 2019, from http://www.depkes.go.id/ article/view/19020100003/hari-kanker-sedunia-2019.html

Molassiotis, A., Yates, P., Li, Q., So, W., Pongthavornkamol, K., Pittayapan, P., ... Si, S. Y. H. (2017). Mapping unmet supportive care needs, quality-of-life perceptions and current symptoms in cancer survivors across the Asia-Pacific region: Results from the International STEP Study. Annals of Oncology, 28(10), 2552-2558. https://doi.org/10.1093/annonc/mdx350

Molassiotis, A, Yates, P., Li, Q., So, W., Pongthavornkamol, K. Pittayapan, P., ... Collaborators $\uparrow$, on behalf of the S. S. (2017). Mapping unmet supportive care needs, quality-of-life perceptions and current symptoms in cancer survivors across the Asia-Pacific region: results from the International STEP Study. Annals of Oncology, 28, 2552-2558. https://doi.org/10.1093/femsec/fix097

Molassiotis, Alex, Yates, P., Li, Q., So, W. K. W., Pongthavornkamol, K., Pittayapan, P., ... Si, S. Y. H. (2017). Mapping unmet supportive care needs, quality-of-life perceptions and current symptoms in cancer survivors across the Asia-Pacific region: Results from the International STEP Study. Annals of Oncology, 28(10), 2552-2558. https://doi.org/10.1093/annonc/mdx350 
National Cancer Institute. (2019). NCI Dictionary. Retrieved from https://www.cancer.gov/publications/dictionaries/cancer-terms/ def/gynecologic-cancer

Nekhlyudov, L., Ganz, P. A., Arora, N. K., \& Rowland, J. H. (2017). Going beyond being lost in transition: A decade of progress in cancer survivorship. Journal of Clinical Oncology, 35(18), 1978-1981. https://doi.org/10.1200/JCO.2016.72.1373

Niksic, M., Rachet, B., Warburton, F. G., Wardle, J., Ramirez, A. J., \& Forbes, L. J. L. (2015). Cancer symptom awareness and barriers to symptomatic presentation in England--are we clear on cancer? British Journal of Cancer, 113(3), 533-542. https://doi.org/10.1038/ bjc. 2015.164

Pangestu, S., \& Karnadi, E. B. (2018). Financial toxicity in Indonesian cancer patients \& survivors: How it affects risk attitude. Cogent Medicine, 00(00), 1-17. https://doi.org/10.1080/23312 05X.2018.1525887

Perwitasari, D. A., Atthobari, J., Dwiprahasto, I., Hakimi, M., Gelderblom, H., Putter, H., ... Kaptein, A. A. (2011). Translation and validation of EORTC QLQ-C30 into Indonesian version for cancer patients in Indonesia. Japanese Journal of Clinical Oncology, 41(4), 519-529. https://doi.org/10.1093/jico/hyq243

Putri, R. H., Afiyanti, Y., Ungsianik, T., \& Milanti, A. (2017). Supportive care needs and quality of life of patients with gynecological cancer undergoing therapy. Enfermería Clínica, 27(Suppl. Part I), 222-226. https://doi.org/doi: 10.1016/S1130-8621(18)30072-X.

Rahman, M. M., Ahsan, M. A., Monalisa, N. N., \& Rahman, K. (2014). Influence of socioeconomic status and BMI on the quality of life after mastectomy in Bangladeshi breast cancer patients in a public hospital. Japanese Journal of Clinical Oncology, 44(12), 1150-1157. https://doi.org/10.1093/jico/hyu144

Ratain, M. J. (2009). Biomarkers and clinical care. AAAS/FDLI Colloquium, Personalized Medicine in an Era of Health Care Reform, Washington, DC.

Rosenberg, S. M., \& Partridge, A. H. (2017). Looking back, moving forward: the evolution of cancer survivorship care. The Lancet Oncology, 18(1), 18-19. https://doi.org/10.1016/ S1470-2045(16)30649-0

Salani, R., Khanna, N., Frimer, M., Bristow, R. E., \& Chen, L. may. (2017). An update on post-treatment surveillance and diagnosis of recurrence in women with gynecologic malignancies: Society of Gynecologic Oncology (SGO) recommendations. Gynecologic Oncology, 146(1), 3-10. https://doi.org/10.1016/j.ygyno.2017.03.022

Schlumbrecht, M., Sun, C., Huang, M., Milbourne, A., \& Bodurka, D. (2018). Gynecologic cancer survivor preferences for long-term surveillance. BMC Cancer, 18(1), 1-10. https://doi.org/10.1186/ s12885-018-4313-x
Schmeler, K. M., Jhingran, A., Iyer, R. B., Sun, C. C., Eifel, P. J., Soliman, P. T., ... Sood, A. K. (2010). Pelvic fractures after radiotherapy for cervical cancer. Cancer, 116(3), 625-630. https:// doi.org/10.1002/cncr.24811

Shih, K. K., Folkert, M. R., Kollmeier, M. A., Abu-Rustum, N. R., Sonoda, Y., Leitao, M. M., ... Alektiar, K. M. (2013). Pelvic insufficiency fractures in patients with cervical and endometrial cancer treated with postoperative pelvic radiation. Gynecologic Oncology, 128(3), 540-543. https://doi.org/10.1016/j. ygyno.2012.12.021

Sleight, A. G., Lyons, K. D., Vigen, C., Macdonald, H., \& Clark, F. (2018). The association of health-related quality of life with unmet supportive care needs and sociodemographic factors in lowincome Latina breast cancer survivors: a single-centre pilot study. Disability and Rehabilitation, O(0), 1-6. https://doi.org/10.1080/0963 8288.2018.1485179

Smith, A., King, M., Butow, P., Luckett, T., Grimison, P., Toner, G., ... Olver, I. (2013). The prevalence and correlates of supportive care needs in testicular cancer survivors: a cross-sectional study. Psychooncology, 22(11), 2557-2564. https://doi.org/10.1002/ pon. 3323

So, W. K. W., Chan, C. W. H., Choi, K. C., Wan, R. W. M., Mak, S. S. S., \& Chair, S. Y. (2013). Perceived unmet needs and health-related quality of life of Chinese cancer survivors at 1 year after treatment. Cancer Nursing, Vol. 36, pp. E23-E32. https://doi.org/DOI: 10.1097/ NCC.0b013e318263f28e Perceived

So, W. K. W., Ming, K. M., Chan, H. Y. L., Chow, K. C., Wan, R. W. M., Mak, S. S. S., ... Chan, C. W. H. (2014). Quality of life and most prevalent unmet needs of Chinese breast cancer survivors at one year after cancer treatment. European Journal of Oncology Nursing, 18(3), 323-328. https://doi.org/10.1016/j.ejon.2014.03.002

Tangcharoensathien, V., Patcharanarumol, W., Ir, P., Aljunid, S., Mukti, A., \& Akkhavong, K, et al. (2011). Health-financing reforms in southeast Asia: challenges in achieving universal coverage. The Lancet, 377, 863-73.

Urbaniec, O. A., Collins, K., Denson, L. A., \& Whitford, H. S. (2011). Gynecological cancer survivors: Assessment of psychological distress and unmet supportive care needs. Journal of Psychosocial Oncology, Vol. 29, pp. 534-551. https://doi.org/DOI: 10.1080/07347332.2011.599829

Yabroff, K. R., Lawrence, W. F., Clauser, S., Davis, W. W., \& Brown, M. L. (2004). Burden of illness in cancer survivors: findings from a population-based national sample. Journal of the National Cancer Institute, 96(17), 1322-1330. https://doi.org/10.1093/jnci/djh255 